\title{
Environmental Consistency Modulation of Error Sensitivity During Motor Adaptation is Explicitly Controlled
}

Guy Avraham ${ }^{1,2,3,4}$, Matan Keizman², and Lior Shmuelof $f^{1,5,6}$

1. Department of Brain and Cognitive Sciences, Ben-Gurion University of the Negev, Be'er Sheva, Israel, 8410501

2. Department of Biomedical Engineering, Ben-Gurion University of the Negev, Be'er Sheva, Israel, 8410501

3. Department of Psychology, University of California, Berkeley, Berkeley, CA, USA, 94720

4. Helen Wills Neuroscience Institute, University of California, Berkeley, Berkeley, CA, USA, 94720

5. Department of Physiology and Cell Biology, Ben-Gurion University of the Negev, Be'er Sheva, Israel, 8410501

6. Zlotowski Center for Neuroscience, Ben-Gurion University of the Negev, Be'er Sheva, Israel, 8410501

Corresponding author: Guy Avraham- guyavraham@berkeley.edu 


\section{Abstract}

Motor adaptation, the adjustment of sensorimotor representations in face of changes in the environment, may operate at different rates. When human participants encounter repeated or consistent perturbations, their corrections for the experienced errors are larger compared to when the perturbations are new or inconsistent. Such modulations of error sensitivity were traditionally considered to be an implicit process that does not require attentional resources. In recent years, the implicit view of motor

7 adaptation is challenged by evidence showing a contribution of explicit strategies to learning. These

8 findings raise a fundamental question regarding the nature of the error sensitivity modulation processes.

9 We tested the effect of explicit control on error sensitivity in a series of experiments, in which participants

10 controlled a screen cursor to virtual targets. We manipulated environmental consistency by presenting

11 rotations in random (low consistency) or random walk (high consistency) sequences, and illustrated that

12 perturbation consistency affects the rate of adaptation, corroborating previous studies. When

13 participants were instructed to ignore the cursor and move directly to the target, thus, eliminating the

14 contribution of explicit strategies, consistency-driven error sensitivity modulation was abolished. In

15 addition, delaying the visual feedback, a manipulation that affects implicit learning, did not influence error

16 sensitivity under consistent perturbations. These results suggest that increases of learning rate in

17 consistent environments are attributable to an explicit rather than implicit process in sensorimotor 18 adaptation.

\section{Significant Statement}

21 When experiencing an error in a motor task (e.g., missing a basketball shot in a windy day), the motor

22 system modifies its next action based on environmental consistency (how frequent the changes in wind's

23 direction and strength are). It is unknown whether this process is driven by an implicit and automatic 
process, or by an explicit process that employs cognitive strategies. We examined these possibilities in a

25 simple visuomotor task by perturbing the feedback in each trial with different consistency levels, and manipulating the use of implicit and explicit processes. We found that participants increase their sensitivity to errors in consistent environments when employing explicit strategies, and do not change their behavior when the implicit process is operating alone.

\section{Introduction}

A fundamental principle in motor learning is modularity. Even simple motor learning behaviors, like adaptation, are driven by multiple learning processes (Smith et al., 2006). A striking behavioral account for modularity in sensorimotor adaptation was demonstrated by the existence of implicit and explicit processes (Mazzoni and Krakauer, 2006; Taylor and Ivry, 2011; Haith and Krakauer, 2013; Taylor et al., 2014). The Implicit learning process refers to an automatic recalibration of the motor response to an experienced error, whereas the explicit learning process is the intentional update of aiming direction following a strategy to improve performance. These processes are also thought to be associated with different neural substrates; implicit learning depends on the cerebellum (Imamizu et al., 2000; Taylor et al., 2010; Galea et al., 2011; Schlerf et al., 2012; Kim et al., 2015; Morehead et al., 2017) whereas explicit learning is associated with cortical function (Taylor and Ivry, 2014; McDougle et al., 2016) and the dopaminergic system (Leow et al., 2012).

Despite the accumulation of results supporting this modularity, the functional roles of the implicit and explicit processes, and the interaction between them during adaptation, are still largely unknown. It was proposed that each learning process is driven by a different error signal; the implicit process is driven by sensory prediction errors, the difference between the expected and the actual sensory feedback, and the 
and Krakauer, 2006; Taylor and Ivry, 2011; Shmuelof et al., 2012a; Reichenthal et al., 2016). This idea can explain the parallel operation of these processes during the time course of visuomotor adaptation (Taylor et al., 2014). However, it does not explain secondary influences on learning, such as modulations of error sensitivity - the change in the reaction to errors - that were reported for different error magnitudes

51 (Criscimagna-Hemminger et al., 2010; Marko et al., 2012) and for different perturbation consistencies

52 (Herzfeld et al., 2014).

Error sensitivity was shown to increase for small errors (Marko et al., 2012). However, this dependency is

54 challenged by recent evidence showing invariance of the implicit process to error magnitude, i.e., that different errors lead to a constant and signed motor correction (Bond and Taylor, 2015; Morehead et al., 2017; Kim et al., 2018). The apparent error-sized dependent modulation of error sensitivity may therefore

57 be an outcome of dividing the (fixed) correction by different error magnitudes.

Another contextual effect on error sensitivity is the consistency of the perturbation. Gonzalez Castro et al., 2014 and Herzfeld et al., 2014 have shown that humans adapt faster to perturbations that are consistent compared to perturbations that are random (Gonzalez Castro et al., 2014; Herzfeld et al., 2014). Importantly, sensitivity to error in these studies was measured for probe trials in which the experienced error was similar across the different consistency conditions, thereby controlling for the concern that modulation of error sensitivity was merely a normalization artifact that reflects differences in error magnitudes. The effect of consistency on adaptation pose an important question regarding its underlying mechanism; on the one hand, the increased learning rate for the consistent errors could be a result of an implicit error-sensitivity modulation in the cerebellum (Herzfeld et al., 2014; Hanajima et al., 

and perturbation's consistency. We report that the modulation of error sensitivity due to the consistency

71 of the perturbation depends on explicit processes, and that manipulating implicit learning has no

72 detectable effect on error sensitivity under consistent perturbations. Our results provide further support

73 for the lack of contextual effects on implicit learning, and emphasize the crucial role of explicit control in 74 sensorimotor learning. conducted after the participants signed an informed consent form approved by the Human Subjects Research Committee of Ben-Gurion University of the Negev, Be'er-Sheva, Israel.

84 Participants sat facing a computer monitor (resolution- $1280 \times 1024$ pixels, dimensions: $37.7 \times 30.1 \mathrm{~cm}$ ), distant by $\sim 1.5$ meters, and controlled a screen cursor by making pointing movements with the fist through flexion-extension and pronation-supination of the right wrist (Krakauer et al., 2006; Shmuelof et

87 al., 2012b). Their right forearm rested within a stabilization device that prevented its supination. The cursor location on the screen was mapped to the position of a retroreflective marker attached to the 
deviation of the cursor. The marker position was recorded at $100 \mathrm{~Hz}$ using three motion capture cameras

91 (Qualisys AB, Sweden).

92 In all experiments, participants were requested to move the cursor to the target by performing a wrist

93 pointing movement (Fig. 1). The start location, depicted as a circle in the center of the screen, and a gray

94 target, $1.2 \mathrm{~cm}$ diameter and distant by $6.3 \mathrm{~cm}$ from the start location, were both presented on the screen

throughout the trial. Each trial was initiated with the appearance of an orange cursor, $0.6 \mathrm{~cm}$ diameter,

simultaneously with a presentation of a tone, signaling the participants to move to a start location, which

was colored in blue. If participants remained in the start location for $1 \mathrm{sec}$, they received a 'Go' cue - both

cursor and start location turned black, marking the onset of a fast out-and back movement with the goal

of placing the reversal point on the target. To eliminate online feedback corrections, the cursor

'Go' cue, participants received performance feedback: the black cursor reappeared at the reversal point

when the center of the cursor was distant by less than $0.65 \mathrm{~cm}$ from the center of the target (i.e., when

107 with respect to movement direction. In Error Clamp trials, the cursor landed in a position that was non- 


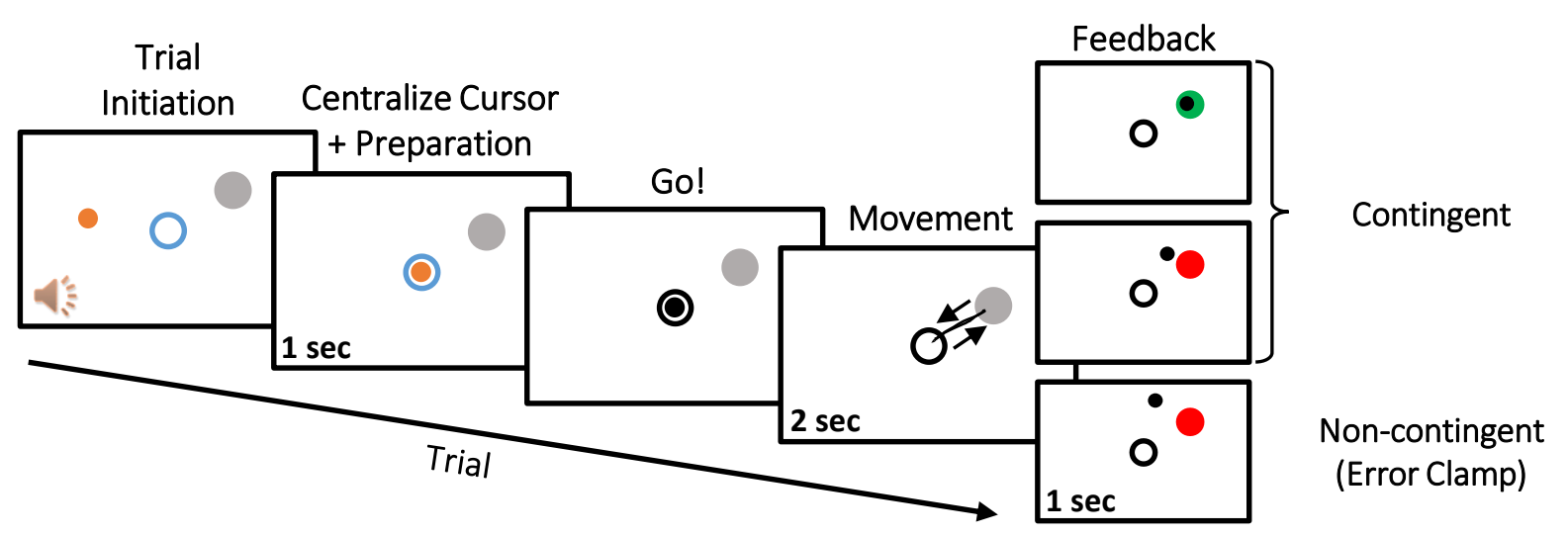

114 Trial initiation was marked by the appearance of a cursor (orange dot) and a presentation of an auditory tone. Participants were requested to move the cursor to a start location (blue circle). Following $1 \mathrm{sec}$ in the start location, they received a 'Go' cue- both cursor and start locations turned black, signaling

117 participants to move to the target (filled gray circle). Participants were requested to perform a fast out118 and-back movement, placing the reversal point on the target. The cursor was not presented during the 119 movement. Movement directions (arrows) and an example of a movement path (black line) are 120 schematically illustrated and were not presented to the participants. Trials ended with participants 121 receiving performance feedback: a black dot reappeared at the reversal point and the target changed 122 colored either to green for target hits, or to red for misses. In Contingent trials, the location of the

123 feedback cursor was contingent on participants' movements, and was either veridical or rotated with 124 respect to the movement direction. In Non-contingent (Error Clamp) trials, the cursor landed at the radius 125 of the target in a predetermined position that was rotated by $15^{\circ}$ clockwise or counterclockwise with 126 respect to the target. 
In all three experiments, participants did multiple experimental runs. Each run started with a baseline epoch with veridical visual feedback. This epoch was followed by an adaptation epoch in which the cursor was rotated with respect to the movement direction of the hand. Rotation magnitudes ranged from $-30^{\circ}$ to $30^{\circ}$ in steps of $5^{\circ}$ (negative and positive values represent counterclockwise and clockwise, respectively). Runs were different by the schedule of the presented rotations, and each run comprised of one of two types of conditions that varied by consistency: Random and Random Walk (see Fig. 2 for illustration of each type). For the Random condition, the rotations were presented in a pseudorandom order, and changed between successive trials, such that the consistency, measured by lag-1 autocorrelation

137 (Gonzalez Castro et al., 2014), is small (Table 1). For the Random Walk condition, the rotations varied from trial to trial according to a random walk algorithm: for each successive trial, the rotation changed by $5^{0}$, either clockwise or counterclockwise. The resulting perturbation function of the Random Walk condition had higher consistency than the Random perturbation function (Table 1).

\begin{tabular}{|c|c|c|}
\hline & Random & Random Walk \\
\hline Experiment 1 & $0.25,0.27,0.18,0.26$ & $0.91,0.91,0.92,0.91$ \\
\hline Experiment 2 & 0.04 & 0.93 \\
\hline Experiment 3 & & $0.91,0.92,0.89,0.93$ \\
\hline
\end{tabular}

143 Table 1. lag-1 autocorrelation $(R(1))$ values. A single value represents the $R(1)$ of a perturbation

144 schedule in a single run, i.e., the correlation coefficient between the rotation magnitudes in two 145 successive trials. All values within each cell represent all possible runs in an experiment. 
147 Most of the trials during the adaptation epoch were Contingent trials and some trials were Error Clamp

148 trials (see below for exact percentages in each experiment). For the Random condition, these two types

149 of trials were presented in pseudorandom and predetermined order. For the Random Walk conditions,

150 the Error Clamp trials were only presented after contingent trials that constitutes of rotation size that was

151 similar to the clamp size $\left(15^{\circ}\right)$, i.e., $10^{0}-20^{\circ}$; the purpose of this design was to avoid disruption of the high

152 consistency of the Random Walk perturbation schedule.

155 Experiment 1 consisted of a single group of participants $(\mathrm{N}=18)$. All participants did four experimental 156 runs. In each run, they moved to a different target that was presented in one of four locations: $45^{\circ}, 135^{\circ}$, $157225^{\circ}$ and $315^{\circ}$. Each run started with a baseline epoch of 12 trials, followed by the adaptation epoch that 158 consisted of 120 trials. $~ 17 \%$ of the trials in the adaptation epoch ( 20 trials) were Error Clamp trials. During 159 the adaptation epoch of two of the four runs, for which targets were separated by $180^{\circ}$ from each other, participants experienced Random perturbation sequences, and during the other two runs, they

161 experienced the Random Walk perturbation sequences (for each consistency condition, each participant 162 experienced two of the four $R(1)$ values in the associated cells in Table 1$)$. The two conditions alternated 163 in each experiment, and we counterbalanced the condition that was presented on the first run across 164 participants. Across participants, all targets were associated with both conditions.

167 Experiment 2 consisted of two groups of participants. All participants did two experimental runs, moving 168 to a single target, presented at $45^{\circ}$. In each run, the baseline epoch consisted of 20 trials, and the 
adaptation epoch consisted of 220 trials. 20 trials in the adaptation epoch ( 9\%) were Error Clamp trials.

170 Unlike in Experiment 1, where Error Clamp trials could appear anytime during the adaptation epoch, here

171 they were presented only after the second half of the run (after trial \#120) to ensure that participants had

172 sufficient exposure to the perturbations and their error sensitivity could be adjusted. During the

173 adaptation epoch of one of the two runs, participants experienced the Random perturbation sequence,

174 and during the other run, they experienced the Random Walk perturbation sequence. All participants

175 experienced the same Random and Random Walk perturbation sequences (Table 1). The order of the

176 conditions was counterbalanced across participants.

177 To examine the role of awareness in error sensitivity modulations, participants of one group (Ignore,

$178 \mathrm{~N}=15$ ) were briefed about the perturbation and were requested to ignore the cursor and to move their

179 hand directly to the target. Participants of the other group (Compensate, $\mathrm{N}=16$ ) were not told about the

180 rotation and were instructed to keep trying to hit the target with the cursor. Such instructions were

181 previously shown to control for the contributions of implicit (Ignore group) and explicit (Compensate

182 group) processes in sensorimotor learning (Welch, 1969; Morehead et al., 2017).

Experiment 3 consisted of a single group of participants $(\mathrm{N}=22)$. All participants did four experimental

186 runs. In each run, they moved to a different target that was presented in one of four locations: $45^{\circ}, 135^{\circ}$,

$187225^{\circ}$ and $315^{\circ}$. The order of the targets was the same for all participants. In each run, the baseline epoch

188 consisted of 12 trials, and the adaptation epoch consisted of the 120 trials. 20 trials in the adaptation

189 epoch ( 17\%) were Error Clamp trials. During the adaptation epoch of all four runs, participants

190 experienced Random Walk perturbation sequences (Table 1), and were requested to always try to hit the

191 target with the cursor. 
192

193

194

211 We calculated learning from error for all trials. Error sensitivity was obtained separately for all $15^{0}$ Error

212 Clamp trials and for all trials in which the experienced absolute error was between $10^{\circ}-20^{\circ}$ (Error Clamp 213 and Contingent trials).

Across runs, we manipulated the implicit process by imposing different delays between the moment of movement reversal and the feedback presentation. Within each run, the delay was either constant at $1,000 \mathrm{~ms}$ or $2,000 \mathrm{~ms}$, or varied randomly between $600-1,500 \mathrm{~ms}$ or $1,600-2,500 \mathrm{~ms}$ in steps of $100 \mathrm{~ms}$. All participants experienced all four types of delay schedules, but the order was randomized between participants. Across participants, all targets were associated with all delay schedules.

Data analysis

The marker position (attached to the fist) was recorded throughout the experiment and sampled at 60 Hz. It was analyzed offline using a custom-written MATLAB code (The MathWorks, Natick, MA, RRID: SCR 001622). To measure the movement reversal of the marker, for each trial, we identified the first sample $(i)$ in which the movement amplitude was smaller than the previous sample $(i-1)$. The movement reversal was defined as the location of the marker on sample $i-1$. We defined the hand's movement angle $(M A)$ as the angle between the imaginary lines connecting the movement origin to the movement reversal and to the target. We calculated the directional error $\left(e_{n}\right)$ at trial $n$ as the angular difference between the feedback and the target. For each error, we measured learning from error $(L E)$, as the change in $M A$ from the trial in which the error was experienced to the next trials:

(1) $L E_{n}=M A_{n+1}-M A_{n}$ Error sensitivity $(E S)$ was calculated as:

(2) $E S_{n}=L E_{n} / e_{n}$ 
214 Learning from error functions were obtained by sorting errors experienced in each condition for each

215 participant based on magnitude, binning them to 12 bins, and averaging the experienced errors and the

216 change in hand angle within each bin. Then, we calculated the between-participant mean and standard

217 error of the experienced errors and the change in hand angle for each bin.

218 We quantified the learning from error by fitting a linear function to the change in hand angle with respect

219 to the error size for each participant and condition. We used the slope of the fitted linear function as a

220 measure for learning from error across all of the experienced error magnitudes. Greater absolute slope

221 values indicate higher error sensitivity.

224 Statistical analyses were performed using custom-written MATLAB functions, the MATLAB Statistics

225 Toolbox, and IBM SPSS (RRID: SCR 002865). We used the Lilliefors test to determine whether our

226 measurements were distributed normally (Lilliefors, 1967). In the repeated-measures ANOVA models, we

227 used Mauchly's test to examine whether the assumption of sphericity was met. For the factors that were

228 statistically significant, we performed planned comparisons and corrected for familywise error using the

229 Bonfferoni correction. We denote the Bonfferoni-corrected $p$ values as $p_{B}$.

230 The statistical analyses for all three experiments were done on the following measures: (1) mean error

231 sensitivity across all $15^{\circ}$ Error Clamp trials, (2) all $10^{\circ}-20^{\circ}$ error trials, and (3) the slope of the learning from

232 error function, calculated separately for each participant and each condition.

233 To examine the influence of consistency on each of the above error sensitivity measures in Experiment 1,

234 for each participant, we pulled together the data from two runs of the same consistency condition

235 (Random and Random Walk), and calculated each participant's error sensitivity measures for each 
consistency condition. We used a two-tail paired-sample $t$ test to examine whether the difference in each

237 measure between the Random and the Random Walk conditions are statistically significant.

238 To examine the effects of explicit strategies on error sensitivity modulation in environment with different

239 levels of consistency in Experiment 2, we calculated error sensitivity measures for each consistency

240 condition. For each measure, we fitted a two-way mixed-effect ANOVA model, with the measure as the

241 dependent variable, one between-participants independent factor (Strategy: two levels, Ignore and

242 Compensate), and one within-participant independent factor (Consistency: two levels, Random and

243 Random Walk).

244 To examine the effects of delayed feedback (modulation of implicit adaptation) on error sensitivity, we

245 calculated each participant's error sensitivity measures for each delay condition. For each measure, we

246 fitted a four-way repeated-measures ANOVA model, with the measure as the dependent variable, and

247 one within-participant independent factor (Delay: four levels, 600-1,500, 1,000, 1,600-2,500, and 2,000

$248 \mathrm{~ms})$.

249 Throughout this paper, statistical significance was set at the $p<0.05$ threshold.

251 Results

252 Experiment 1: Consistency of the perturbation increases error sensitivity

253 A group of participants $(\mathrm{N}=18)$ experienced both Random and Random Walk schedules of visuomotor

254 rotations on different experimental runs (Fig. 2). During the Random condition, the rotations were

255 presented in a pseudorandom order, changing between successive trials, such that the consistency,

256 measured by lag-1 autocorrelation (Gonzalez Castro et al., 2014), is small (mean $R(1)=\sim 0.24$, see

257 methods for further details). During the Random Walk condition, the rotations varied from trial to trial 

(mean $R(1)=\sim 0.91)$ than the Random perturbation function.

\section{a Random}

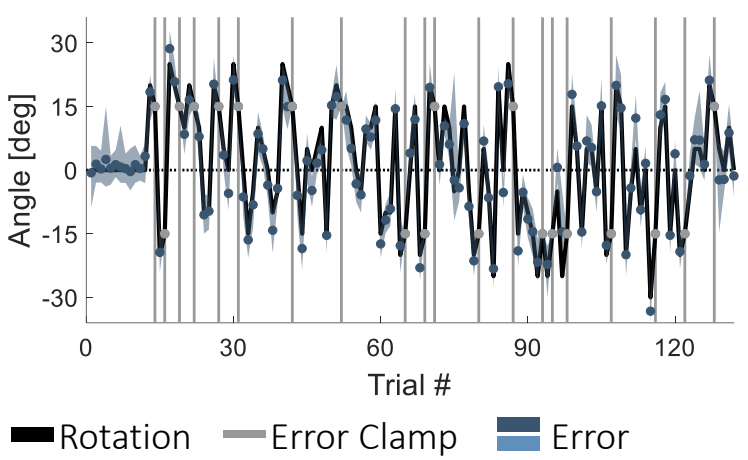

b Random Walk

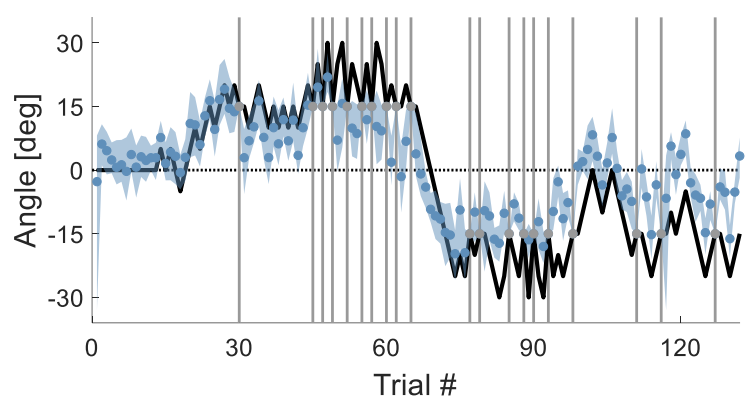

Figure 2. Experiment 1: Angular errors during Random and Random Walk sequences of visuomotor

rotation.

264 Time courses of the applied rotation (black lines) and errors (colored dots) during Random ( $\boldsymbol{a}$, dark blue) and Random Walk ( $\boldsymbol{b}$, light blue) perturbation sequences. Gray dots and vertical lines represent Error Clamp trials. Shading represents $95 \%$ confidence interval. to the Random Walk perturbation; this is evident by the gradual decrease with respect to the rotation size, especially during the late stage of the adaptation epoch (Fig. 2b). The Random perturbation masks

271 any improvement in performance (Fig. 2a).

272 During the Random Walk condition, participants showed higher sensitivity to errors than during the

273 Random condition. To measure error sensitivity, we normalized the change in hand angle by the error size. For Error Clamp trials (trials in which the feedback cursor was presented $15^{\circ}$ away from the target, 
275 irrespective of the performance of the participant), despite the apparent increase in error sensitivity for

276 the random walk group, this effect did not reach significance $[t(17)=1.40, p=0.179$, Fig. 3a]. However,

277 computing error sensitivity for all the trials in which the experienced error size was within a range of $10^{\circ}-$

$27820^{\circ}$, which allowed a more robust estimation of error sensitivity within each participant, reveals a higher

279 sensitivity to these errors in the Random Walk condition than in the Random condition $[t(17)=2.66, p=$

280 0.016, Fig. 3b]. We also examined the trial-by-trial change in hand angle as a function of the error size

281 across the entire range of the experienced errors (learning from error, Fig. 3c). This analysis reveals that

282 the absolute slope of the learning from error function for the Random Walk schedule is higher than for

283 the Random perturbation $[t(17)=2.88, p=0.010]$, suggesting that participants apply bigger

284 corrections for the experienced error during the former than the latter condition. Additionally,

285 participants experienced smaller errors during Random Walk than during Random conditions. The

286 projections of the curves on the abscissa indicate that the errors experienced during the Random Walk

287 condition has a narrower distribution than the errors experienced during the Random condition (as a

288 result of the increased error sensitivity and the consistent perturbation schedule that allowed for a

289 gradual reduction in error size). These results are in agreement with the previous reports that sensitivity

290 to errors in sensorimotor tasks is higher as the consistency in the environment increases (Gonzalez Castro

291 et al., 2014; Herzfeld et al., 2014). 

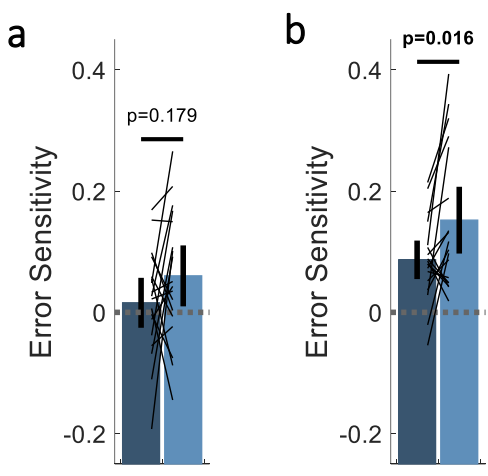

C

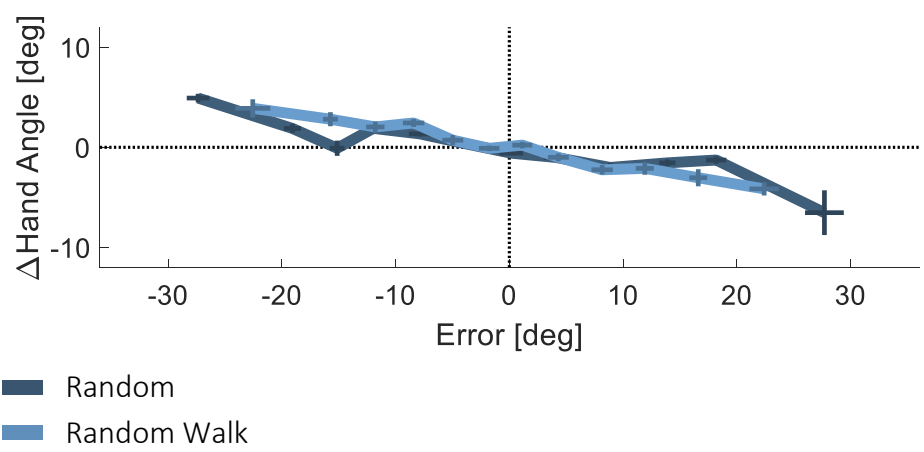

Figure 3. Experiment 1: Error sensitivity is higher for Random Walk than for Random sequences of visuomotor rotation.

$(\boldsymbol{a}, \boldsymbol{b})$ Between-participants mean error sensitivity for each of the Random (dark blue) and Random Walk (light blue) conditions, computed for Error Clamp trials (15 errors, $\boldsymbol{a})$ and for $10^{\circ}-20^{\circ}$ error trials $(\boldsymbol{b})$. Error bars represent $95 \%$ confidence interval. Thin black lines represent data of individual participants. Significant $p$-value is bolded. (c) Mean change in hand angle as a function of the experienced error for the Random and Random Walk perturbation sequences. The trials were binned by error size for each participant. The centers of the crosses represent the mean hand angle for the mean error of each bin, and the vertical and horizontal lines of the crosses represent between-participant standard error.

Experiment 2: Increased error sensitivity in consistent environments depends on explicit strategies

The higher sensitivity to errors for the Random Walk condition with respect to the Random condition in explicit control strategies, or both. We examined these possibilities in Experiment 2. experienced both Random $(R(1)=0.04)$ and Random Walk $(R(1)=0.93)$ perturbation schedules in 
different experimental runs. To examine the role of awareness in error sensitivity modulations, participants in the Ignore group ( $\mathrm{N}=15)$ were requested to ignore the feedback and to move their hand directly to the target, whereas participants in the Compensate group $(\mathrm{N}=16)$ were instructed to keep trying to reach the target with the cursor.

314 Error sensitivity was the highest when participants were explicitly compensating for the Random Walk 315 perturbation schedule. Statistical analyses of error sensitivity in the Error Clamp trials (15 ${ }^{0}$ errors, Fig. 4a) 316 and from the $10^{\circ}-20^{\circ}$ error trials (Fig. 4b) revealed a significantly higher error sensitivity in the 317 Compensate than in the Ignore group (Strategy main effect: $15^{0}$ errors- $F(1,29)=7.01, p=0.013 ; 10^{\circ}$ $20^{0}$ errors- $\left.F(1,29)=19.15, p=1.43 \times 10^{-4}\right)$, suggesting that the Ignore manipulation suppressed error corrections. Similar to Experiment 1, the participants increased their sensitivity to errors when faced with Random Walk rather than Random perturbation schedule in the $10^{\circ}-20^{\circ}$ error trials (Consistency main effect: $15^{0}$ errors- $F(1,29)=2.02, p=0.166 ; 10^{0}-20^{0}$ errors- $\left.F(1,29)=12.20, p=0.002\right)$. Most

322 importantly, we found an interaction effect between strategy and consistency influences on error 323 sensitivity modulation (Strategy-Consistency interaction effect: $15^{0}$ errors- $F(1,29)=5.58, p=0.025$;

$32410^{0}-20^{0}$ errors- $\left.F(1,29)=5.95, p=0.021\right)$. While the Ignore group did not exhibit any change in error 325 sensitivity between the Random and Random Walk conditions ( $15^{\circ}$ errors- $p_{B}=0.518 ; 10^{\circ}-20^{\circ}$ errors$\left.326 p_{B}=0.469\right)$, the Compensate group had a significantly higher error sensitivity during the Random Walk 327 condition than during Random condition ( $15^{0}$ errors- $p_{B}=0.011 ; 10^{0}-20^{0}$ errors- $\left.p_{B}=1.95 \times 10^{-4}\right)$. In 328 addition, a comparison between the groups during the Random Walk condition revealed a significantly 329 higher error sensitivity in the Compensate group with respect to the Ignore group $\left(15^{\circ}\right.$ errors- $p_{B}=0.007$; $10^{0}-20^{0}$ errors- $\left.p_{B}=2.66 \times 10^{-4}\right)$. 

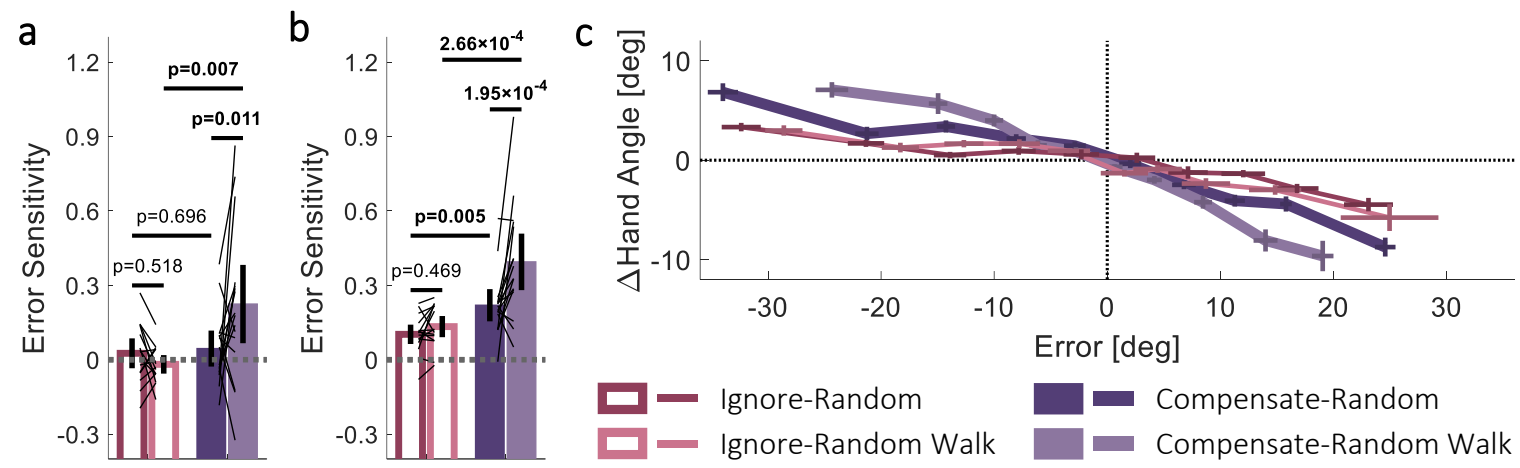

Figure 4. Experiment 2: Error sensitivity is higher for Random Walk than for Random sequences of visuomotor rotation when participants compensate rather than ignore the perturbations. between-participant standard error.

The essential role of explicit strategies on error sensitivity modulation in consistent environments is also magnitudes (Fig. 4c). The absolute slopes of the learning from error functions were significantly higher in the Compensate than in the Ignore group (Strategy main effect: $F(1,29)=23.75, p=3.60 \times 10^{-5}$ ), and during the Random Walk than the Random condition (Consistency main effect: $F(1,29)=20.29, p=$ 
$\left.1.00 \times 10^{-4}\right)$. The difference between the groups increased when they experienced Random Walk compared to Random perturbation schedules (Strategy-Consistency interaction effect: $F(1,29)=$ comparable between the Random and Random Walk conditions $\left(p_{B}=0.344\right)$, the learning from error

354 function of the Compensate-Random Walk condition has a larger absolute slope than the slope of the

357 indicating that during this condition, participants experienced the smallest distribution of errors. Overall, these results suggest that the increase in error sensitivity in consistent environments depends on the use of an explicit strategy.

362 The observed enhancement of error sensitivity in the Random Walk condition in the Compensate group sensitivity in consistent environments. In addition, the absence of difference in error sensitivity between the consistency conditions in the Ignore group suggests that implicit processes do not contribute to changes in error sensitivity. We verified the latter conclusion in Experiment 3.

367 One group of participants $(\mathrm{N}=22)$ experienced Random Walk perturbation schedules across different experimental runs $(R(1)=\sim 0.91)$. The participants were requested to always try to hit the target with the cursor. Across runs, we manipulated the implicit process by imposing different delays between the movement and the feedback. Delayed feedback was previously shown to attenuate adaptation (Kitazawa 
372 or variable $(600-1,500 \mathrm{~ms}$ or $1,600-2,500 \mathrm{~ms})$.

374 addition, during all four delay conditions, participants exhibited similar learning from error functions that does not affect error sensitivity, and therefore provide further support for the sole contribution of explicit control to enhanced error sensitivity in consistent environments.
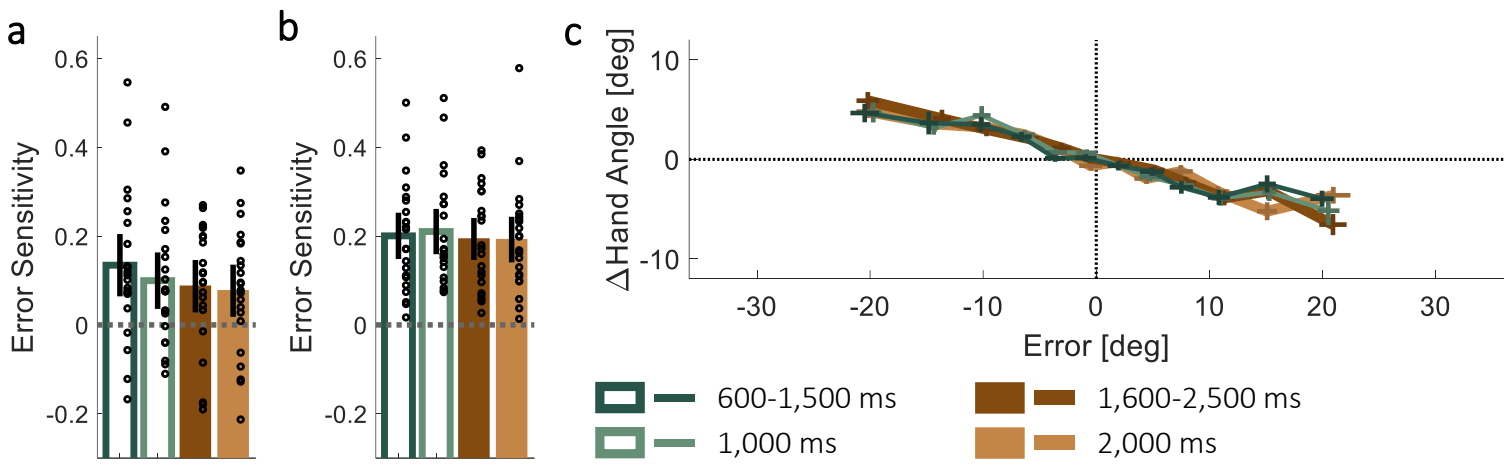

Figure 5. Experiment 3: Error sensitivity for Random Walk is comparable between different magnitudes

$(\boldsymbol{a}, \boldsymbol{b})$ Between-participants mean error sensitivity for feedback delays of $600-1,500 \mathrm{~ms}$ (hollowed dark green bar), 1,000 ms (hollowed light green bar), 1,600-2,500 ms (filled dark brown bar) and 2,000 ms (filled light brown bar), computed for Error Clamp trials ( $15^{\circ}$ errors, $\boldsymbol{a}$ ) and for $10^{\circ}-20^{\circ}$ error trials (b). Error 
bars represent $95 \%$ confidence interval. Black circles represent data of individual participants. (c) Mean change in hand angle as a function of the experienced error for each of the short (thin lines) and long (thick lines) delay conditions. The trials were binned by error size for each participant. The centers of the crosses represent the mean hand angle for the mean error of each bin, and the vertical and horizontal lines of the crosses represent between-participant standard error.

\section{Discussion}

Environmental consistency is considered to be an important factor in sensorimotor learning (Burge et al., random walk sequences of visuomotor perturbations compared to random sequences. Nevertheless, we show that consistency by itself is not a sufficient condition for increasing learning rates. When instructed to ignore the perturbations, a manipulation that suppresses explicit processes and thereby reveal the contribution of implicit learning processes (Mazzoni and Krakauer, 2006; Morehead et al., 2017), our participants did not exhibit an increase in error sensitivity in a consistent environment. Furthermore, variation of the delay of the feedback, a manipulation that typically influences implicit adaptation, yielded 404 comparable levels of sensitivity to errors under random walk conditions, supporting the contribution of explicit learning processes to adaptation in consistent environments. Overall, our results suggest that the sensorimotor system increases error sensitivity in consistent environments due to the contribution of explicit strategies rather than by influencing the rate by which internal models are implicitly updated. conscious awareness of the perturbations. Nevertheless, recent evidence illustrated that the sensitivity

410 function of the explicit learning process to different magnitudes of visuomotor rotations is influenced by 
monotonic: sensitivity increased from inconsistent to low-consistent perturbations, but decreased as

413 consistency increased farther. Importantly, the sensitivity in Hutter and Taylor, 2018, was examined as a

414 function of the perturbation size rather than the experienced visual error, and the distribution of the latter

415 varies at different levels of consistency (Hutter and Taylor, 2018), which may explain the non-

416 monotonicity behavior of the sensitivity function.

417 The main studies that examined consistency effects on error sensitivity used force field perturbations

418 (Gonzalez Castro et al., 2014; Herzfeld et al., 2014). While it is difficult to isolate the contribution of explicit

419 strategies to the compensation for force feedback, previous results with this paradigm may address

420 another central question in motor learning: Do the environmental manipulations affect the sensitivity to

421 error directly, or elicit other explicit strategies, such as a recall of a previous successful action? Gonzalez

422 Castro et al., 2014 showed that if participants are suddenly exposed to a perturbation that is opposite to

423 the trained perturbations, they react to it in the same way that they reacted to the trained perturbations,

424 i.e., they apply a negative and inappropriate correction (Gonzalez Castro et al., 2014). This behavior

425 reflects an involvement of a process other than error sensitivity modulation, such as a recall of previous

426 correct responses (Haith and Krakauer, 2014). Our results support that notion by showing that consistency

427 effects are mediated by explicit control.

428 Which strategy underlies the faster adaptation to consistent perturbations? A recent study provided

429 evidence for distinct cognitive strategies during sensorimotor learning of visuomotor rotation: A recall of

430 stimulus-response contingencies and parametric computation of error correction (McDougle and Taylor,

431 2019). The former relates to a fast process that utilizes memories of acquired associations between stimuli

432 and responses, whereas the latter is a process that computes the aiming direction by means of mental

433 rotation based on the experienced target error (Taylor and Ivry, 2011). We speculate that in the context

434 of a constantly changing perturbations, the consistency in the random walk condition may enhance the

435 mental rotation strategy of the participants. Possibly, a prolonged practice with a consistent perturbation 
would enable a caching process of stimulus response associations (Huberdeau et al., 2017) mediated by

437 an increase in movement repetitions (Huang et al., 2011; Mawase et al., 2018).

The link between error sensitivity and explicit sensorimotor learning is also studied in the context of savings, i.e., the faster learning upon re-exposure to the same perturbation. Herzfeld et al., 2014 explained savings as a change in error sensitivity in face of an error that was previously encountered (Herzfeld et al., 2014). More recently, savings was explained as a retrieval of an explicit aiming strategy that was previously associated with a better performance (Haith et al., 2015; Morehead et al., 2015). Interestingly, despite previous evidence for the necessary role of both error-driven adaptation and movement repetitions in

444 inducing savings (Huang et al., 2011), Leow at al., 2016 challenged this view, suggesting that the experience of previously encountered errors is sufficient as long as the perturbation is adequately stable

446 (Leow et al., 2016). Our results are in line with this idea and suggest that savings is a product of enhanced 447 sensitivity to an error that was processes explicitly due to the experience of consistent perturbations. 448 Thus, savings can be viewed as an exemplar for more general phenomena of error correction facilitation 449 that is driven by strategic processes. This conjecture also leads to the prediction that the neural substrates 450 of savings that are localized to the motor cortex (Li et al., 2001; Landi et al., 2011) and basal ganglia circuits 451 (Leow et al., 2012; Ruitenberg et al., 2018) will also be involved in the increased corrections for consistent 452 perturbations. Nonetheless, savings and reaction to consistency may differ in terms of the processes that 453 underlie their formation; while savings possibly depends on encoding of successful reactions to distinct 454 errors (Huang et al., 2011; Huberdeau et al., 2015), consistency detection requires a continuous 455 estimation of the ability to reduce external errors (Baddeley et al., 2003; Gonzalez Castro et al., 2014).

456 The current study supplements the growing body of literature that emphasizes the important contribution 457 of explicit strategies to sensorimotor learning. Whereas the implicit process is highly rigid, the explicit 458 process can be flexibly tuned to task demands, e.g., by scaling the correction to the error size (Bond and 459 Taylor, 2015), and as mentioned above, by adjusting the reactions according to previous experience 
460 (Haith et al., 2015; Morehead et al., 2015; Huberdeau et al., 2017). Furthermore, age-related declines in

461 motor learning were recently shown to be associated with both behavioral and neural changes in the

462 explicit memory system (Vandevoorde and Xivry, 2018; Wolpe et al., 2018). Here we show that interaction

463 between environmental consistency and motor learning is related to the modulations of explicit strategies

464 rather than to the modulation of implicit adaptation.

465 Error clamp trials were previously used to probe changes of sensorimotor representations in face of 466 dynamic (Scheidt et al., 2000; Avraham et al., 2017) and kinematic (Shmuelof et al., 2012a; Morehead et 467 al., 2017) perturbations. In our design, each experimental run consisted of multiple error clamp trials with $46815^{\circ}$ error size, aiming to provide sufficient recurrences for calculating error sensitivity for a constant error.

469 Surprisingly, in some of our experiments, analysis of the error clamp trials yielded no change in error 470 sensitivity (Figs. 3a and 4a). As these trials include feedback that is non-contingent on participants' 471 movements, the fact that they were presented multiple times within a continuously changing 472 environment may have devaluated the relevancy of the associated error size, which in turn was 473 discounted by the motor system (Wei and Körding, 2009). Therefore, we also examined error sensitivity 474 for trials in which the experienced error was similar in size to the error experienced during error clamp 475 trials $\left(10^{\circ}-20^{\circ}\right)$. These analyses revealed that error sensitivity was indeed modulated in all our conditions 476 (e.g., Figs. 3b and 4b). Importantly, the analysis results of the error clamp trials show that error sensitivity 477 is largest under consistent environments and an explicit instruction to compensate for the errors, and 478 thus, they are in line with the main conclusion of this study.

479 The association between consistency and explicit strategies is mediated by the detection of the 480 consistency. Consistency estimation requires monitoring both the perceived errors and the reaction to 481 these errors, but could also be approximated by memorizing the history of error magnitudes alone (by 482 either averaging recent errors or monitoring their trial-by-trial changes). The use of explicit strategies may contribute to the consistency estimation process by increasing the awareness to the error (Johnson et al., 
484 2002), enhancing the notion of agency (Parvin et al., 2018), or facilitating the generation of memories for

485 the experienced errors (Morehead et al., 2015). Understanding the benefits and the driving signals of

486 explicit learning strategies is essential for improving motor learning in general, and specifically, could

487 improve the outcomes of rehabilitative treatments.

References

490 Avraham G, Mawase F, Karniel A, Shmuelof L, Donchin O, Mussa-Ivaldi FA, Nisky I (2017) Representing

491 delayed force feedback as a combination of current and delayed states. Journal of Neurophysiology

$492 \quad 118: 2110-2131$.

493 Baddeley RJ, Ingram HA, Miall RC (2003) System Identification Applied to a Visuomotor Task: Near-

494 Optimal Human Performance in a Noisy Changing Task. J Neurosci 23:3066-3075.

495 Bond KM, Taylor JA (2015) Flexible explicit but rigid implicit learning in a visuomotor adaptation task.

496 Journal of Neurophysiology 113:3836-3849.

497 Brudner SN, Kethidi N, Graeupner D, Ivry RB, Taylor JA (2016) Delayed feedback during sensorimotor

498 learning selectively disrupts adaptation but not strategy use. Journal of Neurophysiology 115:1499-

4991511.

500 Burge J, Ernst MO, Banks MS (2008) The statistical determinants of adaptation rate in human reaching.

501 Journal of Vision 8:20-20.

502 Criscimagna-Hemminger SE, Bastian AJ, Shadmehr R (2010) Size of Error Affects Cerebellar Contributions

503 to Motor Learning. Journal of Neurophysiology 103:2275-2284. 
504 Galea JM, Vazquez A, Pasricha N, Orban de Xivry J-J, Celnik P (2011) Dissociating the Roles of the

505 Cerebellum and Motor Cortex during Adaptive Learning: The Motor Cortex Retains What the Cerebellum

506 Learns. Cereb Cortex 21:1761-1770.

507 Gonzalez Castro LN, Hadjiosif AM, Hemphill MA, Smith MA (2014) Environmental Consistency

508 Determines the Rate of Motor Adaptation. Current Biology 24:1050-1061.

509 Haith AM, Huberdeau DM, Krakauer JW (2015) The Influence of Movement Preparation Time on the

510 Expression of Visuomotor Learning and Savings. J Neurosci 35:5109-5117.

511 Haith AM, Krakauer JW (2013) Model-Based and Model-Free Mechanisms of Human Motor Learning. In:

512 Progress in Motor Control (Richardson MJ, Riley MA, Shockley K, eds), pp 1-21 Advances in Experimental 513 Medicine and Biology. Springer New York.

514 Haith AM, Krakauer JW (2014) Motor Learning: The Great Rate Debate. Current Biology 24:R386-R388.

515 Hanajima R, Shadmehr R, Ohminami S, Tsutsumi R, Shirota Y, Shimizu T, Tanaka N, Terao Y, Tsuji S,

516 Ugawa Y, Uchimura M, Inoue M, Kitazawa S (2015) Modulation of error-sensitivity during a prism

517 adaptation task in people with cerebellar degeneration. Journal of Neurophysiology 114:2460-2471.

518 Herzfeld DJ, Vaswani PA, Marko M, Shadmehr R (2014) A memory of errors in sensorimotor learning.

519 Science:1253138.

520 Huang VS, Haith A, Mazzoni P, Krakauer JW (2011) Rethinking Motor Learning and Savings in Adaptation

521 Paradigms: Model-Free Memory for Successful Actions Combines with Internal Models. Neuron 70:787-

522801.

523 Huberdeau DM, Haith AM, Krakauer JW (2015) Formation of a long-term memory for visuomotor

524 adaptation following only a few trials of practice. Journal of Neurophysiology 114:969-977. 
525 Huberdeau DM, Krakauer JW, Haith AM (2017) Practice induces a qualitative change in the memory

526 representation for visuomotor learning. bioRxiv:226415.

527 Hutter SA, Taylor JA (2018) Relative sensitivity of explicit reaiming and implicit motor adaptation. Journal

528 of Neurophysiology 120:2640-2648.

529 Imamizu H, Miyauchi S, Tamada T, Sasaki Y, Takino R, Pütz B, Yoshioka T, Kawato M (2000) Human

530 cerebellar activity reflecting an acquired internal model of a new tool. Nature 403:192-195.

531 Johnson H, van Beers RJ, Haggard P (2002) Action and awareness in pointing tasks. Exp Brain Res

$532 \quad 146: 451-459$.

533 Kim HE, Morehead JR, Parvin DE, Moazzezi R, Ivry RB (2018) Invariant errors reveal limitations in motor

534 correction rather than constraints on error sensitivity. Communications Biology 1:19.

535 Kim S, Ogawa K, Lv J, Schweighofer N, Imamizu H (2015) Neural Substrates Related to Motor Memory

536 with Multiple Timescales in Sensorimotor Adaptation. PLOS Biology 13:e1002312.

537 Kitazawa S, Kohno T, Uka T (1995) Effects of delayed visual information on the rate and amount of prism

538 adaptation in the human. J Neurosci 15:7644-7652.

539 Krakauer JW, Mazzoni P, Ghazizadeh A, Ravindran R, Shadmehr R (2006) Generalization of Motor

540 Learning Depends on the History of Prior Action. PLOS Biology 4:e316.

541 Landi SM, Baguear F, Della-Maggiore V (2011) One Week of Motor Adaptation Induces Structural

542 Changes in Primary Motor Cortex That Predict Long-Term Memory One Year Later. J Neurosci 31:11808-

54311813. 
544 Leow L-A, de Rugy A, Marinovic W, Riek S, Carroll TJ (2016) Savings for visuomotor adaptation require

545 prior history of error, not prior repetition of successful actions. Journal of Neurophysiology 116:1603-

5461614.

547 Leow L-A, Loftus AM, Hammond GR (2012) Impaired savings despite intact initial learning of motor

548 adaptation in Parkinson's disease. Exp Brain Res 218:295-304.

549 Li C-SR, Padoa-Schioppa C, Bizzi E (2001) Neuronal Correlates of Motor Performance and Motor Learning

550 in the Primary Motor Cortex of Monkeys Adapting to an External Force Field. Neuron 30:593-607.

551 Lilliefors HW (1967) On the Kolmogorov-Smirnov Test for Normality with Mean and Variance Unknown.

552 Journal of the American Statistical Association 62:399-402.

553 Marko MK, Haith AM, Harran MD, Shadmehr R (2012) Sensitivity to prediction error in reach adaptation.

554 Journal of Neurophysiology 108:1752-1763.

555 Mawase F, Lopez D, Celnik PA, Haith AM (2018) Movement Repetition Facilitates Response Preparation.

556 Cell Reports 24:801-808.

557 Mazzoni P, Krakauer JW (2006) An Implicit Plan Overrides an Explicit Strategy during Visuomotor

558 Adaptation. J Neurosci 26:3642-3645.

559 McDougle SD, Ivry RB, Taylor JA (2016) Taking Aim at the Cognitive Side of Learning in Sensorimotor

560 Adaptation Tasks. Trends in Cognitive Sciences 20:535-544.

561 McDougle SD, Taylor JA (2019) Dissociable cognitive strategies for sensorimotor learning. Nature

562 Communications 10:40.

563 Morehead JR, Qasim SE, Crossley MJ, Ivry R (2015) Savings upon Re-Aiming in Visuomotor Adaptation. J

564 Neurosci 35:14386-14396. 
Morehead JR, Taylor JA, Parvin DE, Ivry RB (2017) Characteristics of Implicit Sensorimotor Adaptation

Revealed by Task-irrelevant Clamped Feedback. Journal of Cognitive Neuroscience 29:1061-1074.

567

Parvin DE, McDougle SD, Taylor JA, Ivry RB (2018) Credit assignment in a motor decision making task is

568 influenced by agency and not sensorimotor prediction errors. J Neurosci:3601-3617.

569 Reichenthal M, Avraham G, Karniel A, Shmuelof L (2016) Target size matters: target errors contribute to

570 the generalization of implicit visuomotor learning. Journal of Neurophysiology 116:411-424.

571 Ruitenberg MFL, Koppelmans V, Dios YED, Gadd NE, Wood SJ, Reuter-Lorenz PA, Kofman I, Bloomberg JJ,

572 Mulavara AP, Seidler RD (2018) Neural correlates of multi-day learning and savings in sensorimotor

573 adaptation. Scientific Reports 8:14286.

574 Scheidt RA, Reinkensmeyer DJ, Conditt MA, Rymer WZ, Mussa-Ivaldi FA (2000) Persistence of Motor

575 Adaptation During Constrained, Multi-Joint, Arm Movements. Journal of Neurophysiology 84:853-862.

576 Schlerf J, Ivry RB, Diedrichsen J (2012) Encoding of Sensory Prediction Errors in the Human Cerebellum. J

577 Neurosci 32:4913-4922.

578 Shmuelof L, Huang VS, Haith AM, Delnicki RJ, Mazzoni P, Krakauer JW (2012a) Overcoming Motor

579 "Forgetting" Through Reinforcement Of Learned Actions. J Neurosci 32:14617-14621a.

580 Shmuelof L, Krakauer JW, Mazzoni P (2012b) How is a motor skill learned? Change and invariance at the

581 levels of task success and trajectory control. Journal of Neurophysiology 108:578-594.

582 Smith MA, Ghazizadeh A, Shadmehr R (2006) Interacting Adaptive Processes with Different Timescales

583 Underlie Short-Term Motor Learning. PLOS Biology 4:e179.

584 Taylor JA, Ivry RB (2011) Flexible Cognitive Strategies during Motor Learning. PLOS Computational

585 Biology 7:e1001096. 
586 Taylor JA, Ivry RB (2014) Chapter 9 - Cerebellar and Prefrontal Cortex Contributions to Adaptation,

587 Strategies, and Reinforcement Learning. In: Progress in Brain Research (Ramnani N, ed), pp 217-253

588 Cerebellar Learning. Elsevier. Available at:

589 http://www.sciencedirect.com/science/article/pii/B9780444633569000091 [Accessed July 20, 2018].

590 Taylor JA, Klemfuss NM, Ivry RB (2010) An Explicit Strategy Prevails When the Cerebellum Fails to

591 Compute Movement Errors. Cerebellum 9:580-586.

592 Taylor JA, Krakauer JW, Ivry RB (2014) Explicit and Implicit Contributions to Learning in a Sensorimotor

593 Adaptation Task. J Neurosci 34:3023-3032.

594 Vandevoorde K, Xivry J-JO de (2018) Internal model recalibration does not deteriorate with age while

595 motor adaptation does. bioRxiv:292250.

596 Wei K, Körding K (2009) Relevance of Error: What Drives Motor Adaptation? Journal of Neurophysiology

$597 \quad 101: 655-664$.

598 Welch RB (1969) Adaptation to prism-displaced vision: The importance of target-pointing. Perception \&

599 Psychophysics 5:305-309.

600 Wolpe N, Ingram JN, Tsvetanov KA, Henson RN, Kievit RA, Wolpert DM, Neuroscience CC for A and,

601 Rowe JB (2018) Motor learning decline with age is related to differences in the explicit memory system.

602 bioRxiv:353870.

603

604 Figure Captions

605 Figure 1. Experimental task 
Trial initiation was marked by the appearance of a cursor (orange dot) and a presentation of an auditory

607 tone. Participants were requested to move the cursor to a start location (blue circle). Following 1 sec in

608 the start location, they received a 'Go' cue- both cursor and start location turned black, signaling

609 participants to move to the target (filled gray circle). Participants were requested to perform a fast out-

610 and-back movement, placing the reversal point on the target. The cursor was not presented during the

611 movement. Movement directions (arrows) and an example of a movement path (black line) are

612 schematically illustrated and were not presented to the participants. Trials ended with participants

613 receiving performance feedback: a black dot reappeared at the reversal point and the target changed

614 colored either to green for target hits, or to red for misses. In Contingent trials, the location of the

615 feedback cursor was contingent on participants' movements. In Non-contingent (Error Clamp) trials, the

616 cursor landed at the radius of the target, in a predetermined position that was rotated by $15^{\circ}$ clockwise

617 or counterclockwise with respect to the target.

Figure 2. Experiment 1: Angular errors during Random and Random Walk sequences of visuomotor rotation.

621 Time courses of the applied rotation (black lines) and errors (colored dots) during Random ( $\boldsymbol{a}$, dark blue)

622 and Random Walk ( $b$, light blue) perturbation sequences. Gray dots and vertical lines represent Error

623 Clamp trials. Shading represents 95\% confidence interval. 
$(\boldsymbol{a}, \boldsymbol{b})$ Between-participants mean error sensitivity for each of the Random (dark blue) and Random Walk

628 (light blue) conditions, computed for Error Clamp trials $\left(15^{\circ}\right.$ errors, $\left.\boldsymbol{a}\right)$ and for $10^{\circ}-20^{\circ}$ error trials $(\boldsymbol{b})$. Error bars represent $95 \%$ confidence interval. Thin black lines represent data of individual participants. Significant $p$-value is bolded. (c) Mean change in hand angle as a function of the experienced error for the

631 Random and Random Walk perturbation sequences. The trials were binned by error size for each participant. The centers of the crosses represent the mean hand angle for the mean error of each bin, and the vertical and horizontal lines of the crosses represent between-participant standard error. visuomotor rotation when participants compensate rather than ignore the perturbations.

641 Significant p-values are bolded. (c) Mean change in hand angle as a function of the experienced error for 642 the Ignore (thin lines) and Compensate (thick lines) groups in each type of perturbation sequence. The

643 trials were binned by error size for each participant. The centers of the crosses represent the mean hand 644 angle for the mean error of each bin, and the vertical and horizontal lines of the crosses represent 645 between-participant standard error. 
$649(\boldsymbol{a}, \boldsymbol{b})$ Between-participants mean error sensitivity for feedback delays of $600-1,500$ ms (hollowed dark

650 green bar), 1,000 ms (hollowed light green bar), 1,600-2,500 ms (filled dark brown bar) and 2,000 ms

651 (filled light brown bar), computed for Error Clamp trials $\left(15^{\circ}\right.$ errors, $\left.\boldsymbol{a}\right)$ and for $10^{\circ}-20^{\circ}$ error trials (b). Error

652 bars represent $95 \%$ confidence interval. Black circles represent data of individual participants. (c) Mean

653 change in hand angle as a function of the experienced error for each of the short (thin lines) and long

654 (thick lines) delay conditions. The trials were binned by error size for each participant. The centers of the

655 crosses represent the mean hand angle for the mean error of each bin, and the vertical and horizontal

656 lines of the crosses represent between-participant standard error. 


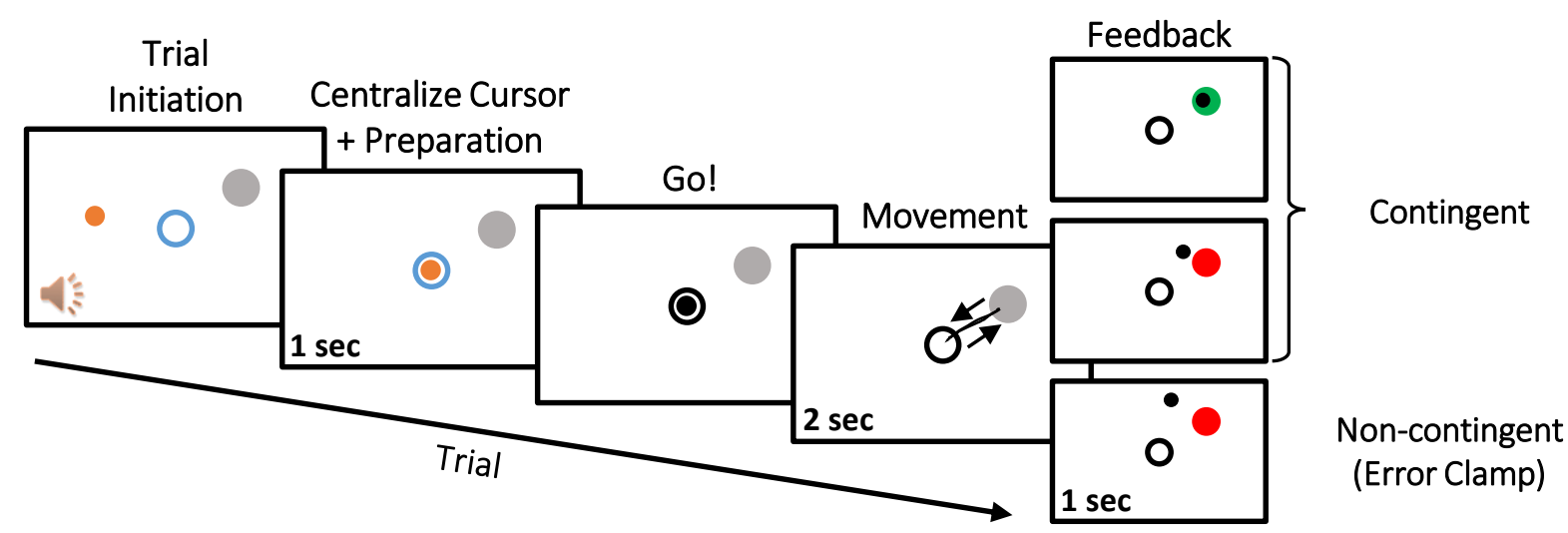


a

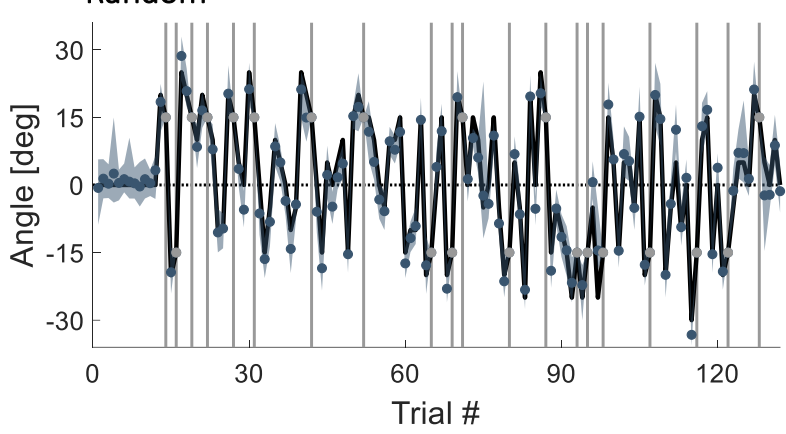

-Rotation - Error Clamp Error b

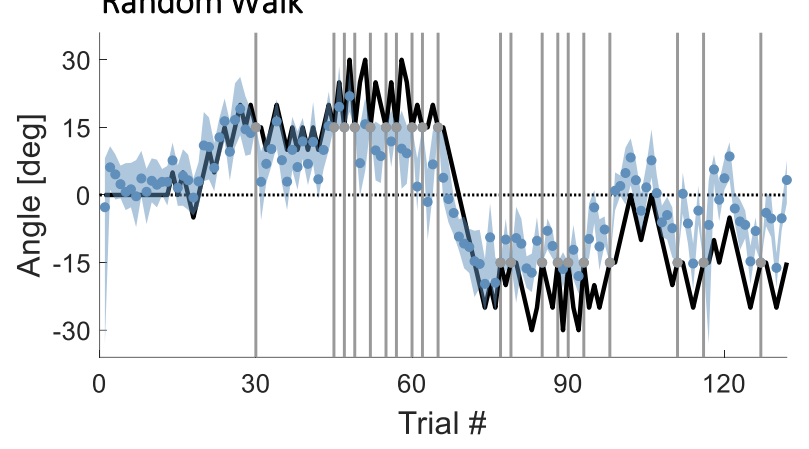



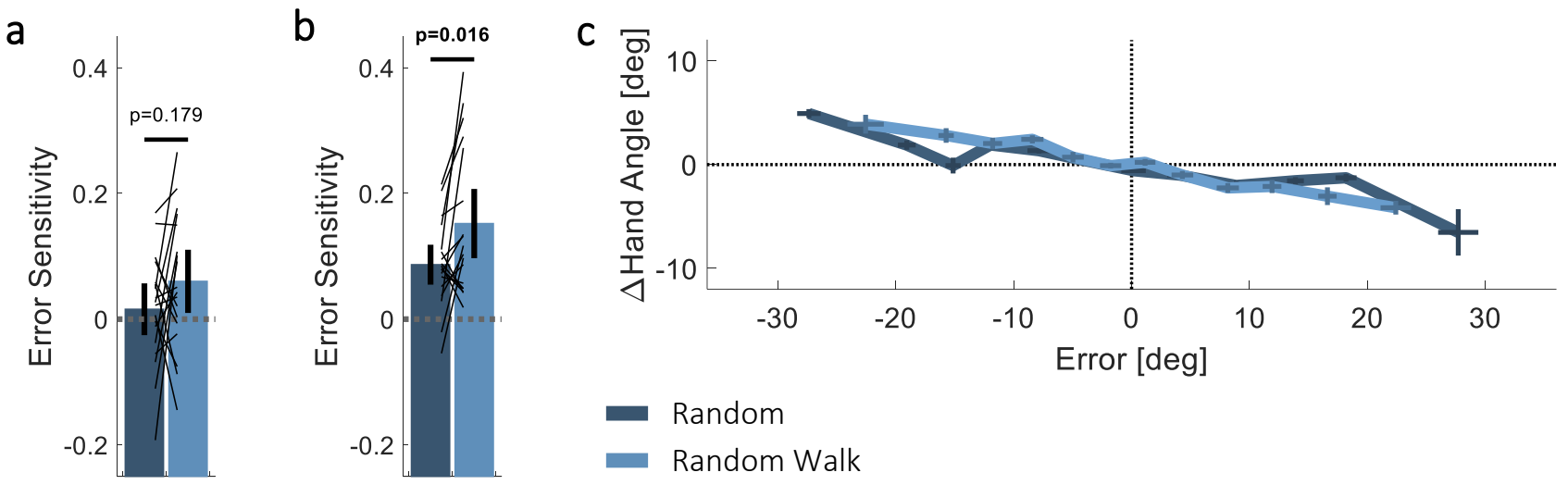

Random
Random Walk 

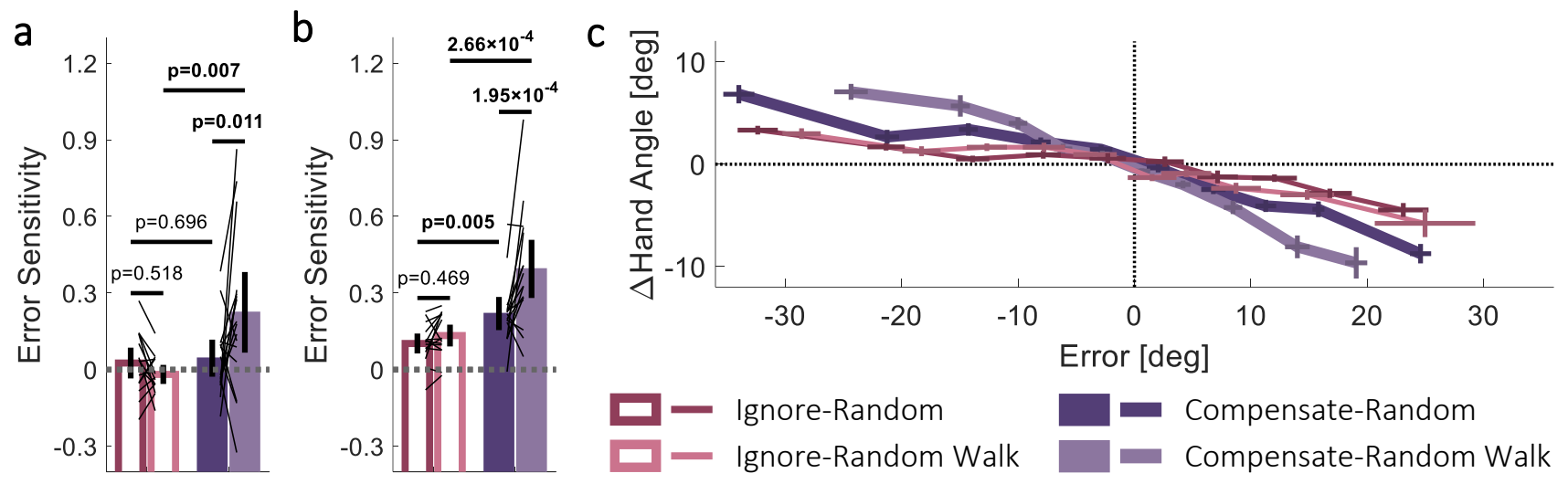

Ignore-Random
Ignore-Random Walk

Compensate-Random

Compensate-Random Walk 

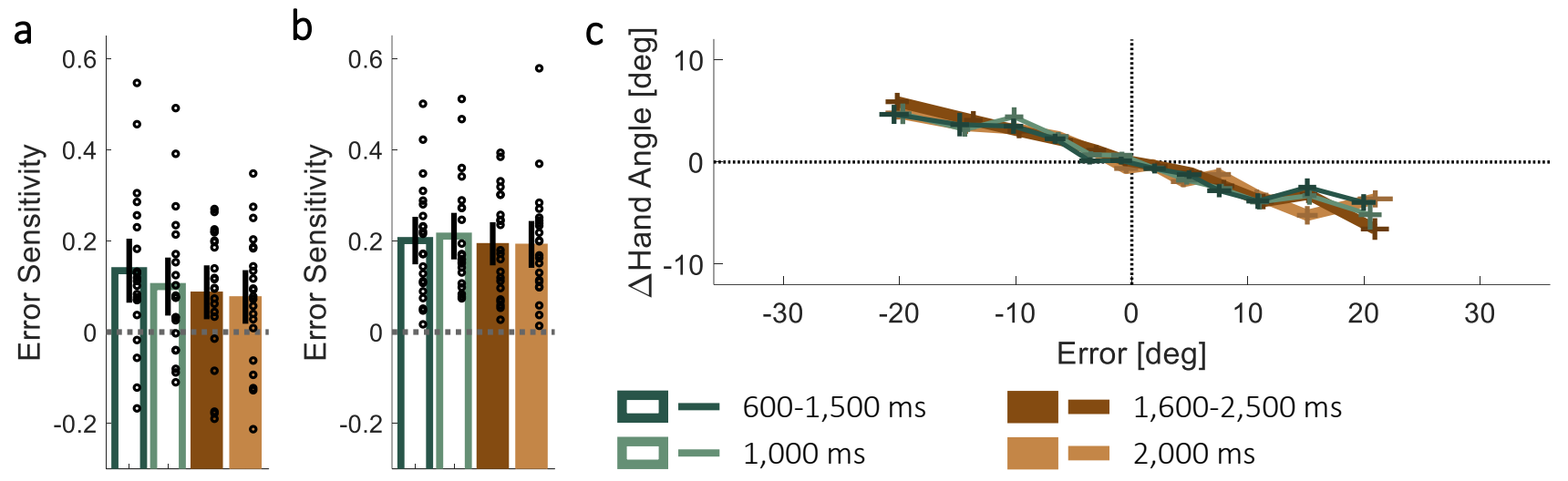